\title{
Computed tomography findings after endoscopic sinus surgery with preserving or enlarging maxillary sinus ostium surgery*
}

\author{
Jyri Myller ${ }^{1,2}$, Prasun Dastidar ${ }^{1,3}$, Tommi Torkkeli, Markus Rautiainen $^{1,5}$, \\ Sanna Toppila-Salmi ${ }^{1,6,7}$ \\ Department of Otorhinolaryngology, University of Tampere, Tampere, Finland \\ Department of Otorhinolaryngology, Päijät-Häme Central Hospital, Lahti, Finland \\ 3 Medical Imaging Centre, Department of Radiology, Tampere University Hospital, Tampere, Finland \\ 4 Department of Otorhinolaryngoly, Mikkeli Central Hospital, Mikkeli, Finland \\ 5 Department of Eye, Ear and Oral diseases, Tampere University Hospital, Tampere, Finland \\ 6 Transplantation laboratory, Haartman Institute, University of Helsinki, Helsinki, Finland \\ 7 Helsinki University Central Hospital, Skin and Allergy Hospital, Helsinki, Finland
}

SUMMARY

\begin{abstract}
Endoscopic sinus surgery (ESS) is the main surgical approach in the treatment of chronic rhinosinusitis (CRS) after failure of medical treatment. ESS is based on the theory that obstruction of the maxillary sinus ostium is mainly behind the pathogenesis of CRS. Controversy remains concerning the enlargement of the natural maxillary sinus ostium. The aim of this study was to compare computed tomography (CT) findings after preservation or enlargement of the maxillary sinus ostium. Thirty patients with non-polypous $C R S$ underwent randomized endoscopic sinus surgery with uncinectomy on one side and additional middle meatal antrostomy on the other side. Lund-Mackay (LM) scores and the ostium diameters were analysed from CT scans taken preoperatively and nine months postoperatively, and were used for comparison of the two operative techniques. In addition, the correlation between CT findings and subjective outcomes was studied. Comparison of the preoperative and postoperative CT scans revealed that significant reduction of LM score was achieved on both sides, regardless of the type of procedure performed. The postoperative area of the ostium remained significantly larger on the antrostomy side compared to the uncinectomy side. A large maxillary sinus ostium size seems to associate with lower postoperative LM score, but does not seem to provide superior symptom relief.
\end{abstract}

Key words: chronic rhinosinusitis, endoscopic sinus surgery, computed tomography, LundMackay score.

\section{INTRODUCTION}

Since its introduction, endoscopic sinus surgery (ESS) has been widely accepted in the treatment of chronic rhinosinusitis (CRS) after failure of medical treatment ${ }^{(1)}$. Reported results have been good both in short- and long-term surveys ${ }^{(2,3)}$.

ESS is based on the theory that the maxillary sinus ostium is the most important area in the pathogenesis of chronic and recurrent rhinosinusitis ${ }^{(15,16)}$. Obstruction of the ostium is believed to lead to chronic inflammation and eventually to pathologic alterations of the maxillary sinus mucosa. Therefore, surgical opening of the ostium and thus improved drainage and ventilation of the sinus should restore the normal mucosa ${ }^{(4)}$.

There are different opinions concerning the extent of surgery of the ostiomeatal complex. It is considered that removal of the uncinate process alone would be enough to restore the ventilation of the maxillary sinus. ESS with the minimally invasive technique aims to achieve normal sinus function and prevent sinus exposure to environmental irritants, by causing minimal opening of the sinonasal structures ${ }^{(5,6)}$.

Despite the fact that the ostium is considered to be the most 
important area in the pathogenesis of CRS, few studies have addressed the role of middle meatal antrostomy in patient symptoms and in objective postoperative findings ${ }^{(7,17,19)}$. Although promising results exist about limited approach of the maxillary sinus ostia, there are no results available concerning the computed tomography outcomes of preservation vs. enlargement of the maxillary sinus ostium ${ }^{(7-9)}$.

The Lund-Mackay (LM) scoring system was developed for objective quantification of the inflammatory disease in the paranasal sinuses ${ }^{(10)}$. It is based on a simple numeric score driven from the CT scan in which every sinus group is assigned a numeric grade depending on the extent of the disease ${ }^{(10)}$. Although CT and endoscopic scores correlate well, the correlation between CT findings and symptom scores has generally shown to be poor and is not a good indicator of the outcomes $(1,11,12)$.

The purpose of this study was to compare preoperative and postoperative CT findings in patients with CRS after uncinectomy with additional middle meatal antrostomy to those after uncinectomy without enlarging the natural ostium. For assessment of the clinical relevance of these results, we also studied correlation between postoperative CT findings and symptoms asked.

\section{MATERIALS AND METHODS}

Subjects

This prospective, randomized, single-blinded study was carried out at the Department of Otorhinolaryngology, Tampere University Hospital, Finland and Mikkeli Central Hospital, Mikkeli, Finland between 2001 and 2003. The ethical committees of the Tampere University Hospital and Mikkeli Central Hospital approved the study. Informed consent was obtained from the patients in this study. Thirty patients having non-polypous CRS, and not responding to maximal medical treatment, were enrolled in this study. They underwent randomly and single-blindly uncinectomy-only on one side and additional middle meatal antrostomy on the contralateral side. The study methods were symptom questionnaire and sinus CT scans performed prior to and nine months after the ESS. Characteristics about the groups of patients are shown in Table 1.

Inclusion criteria were: moderate to severe sinus-related symptoms during at least 12 weeks, despite maximal medical treatment, e.g. at least two major factors (facial pain, nasal obstruction, nasal discharge, hyposmia, and anosmia), or at least one major and two minor factors (fever, halitosis, fatigue, dental pain, cough, and ear pain); endoscopic findings (mucosal thickening, purulent discharge); and total Lund-Mackay sinus computed tomography scores of at least $6 / 24$ but no more than $18 / 24$.

Exclusion criteria were: age less than 18 years; oral corticosteroid treatment during the last two months prior to surgery; previous sinonasal surgery; a history or physical examination suggestive of severe nasal septal deviation (that causes only unilateral nasal obstruction and/or requires septoplasty before performing ESS), unilateral sinusitis, nasal polyposis $>$ grade 1, aspirin sensitivity, chronic bronchitis, cystic fibrosis, tumor or disease with severe impact on general immunity; mild sinusrelated symptoms; and the following computed tomography findings: severe chronic pansinusitis (total opacification in posterior ethmoidal and/or sphenoidal and/or frontal sinuses and/ or total obstruction of frontal recess) and Lund-Mackay score less than $6 / 24$ or more than $18 / 24$.

Dropouts from the study: one patient died accidentally prior to the postoperative control, thus the postoperative CT-scans were not taken.

Diagnosis of nasal polyposis was based on endoscopic examination and pathological evaluation of polyp tissue. Diagnosis of allergic rhinitis was based on skin prick test positivity and at least a two-year history of major symptoms (rhinorrhea, nasal obstruction, nasal itching, and sneezing). Of those who were taking nasal corticosteroids for at least two months before computed tomography scans, 10 had momethasone furoate $50 \mathrm{ug} /$ nostril twice a day (Schering-Plough, Kenilworth, NJ, USA), and 2 had fluticasone proprionate $50 \mathrm{ug} /$ nostril twice a day (Glaxosmithkline, London, UK). Of those who used oral antihistamines with or without oral decongestant for at least 2 weeks before taking biopsies, 1 subject used cetirizine hydrochloride $10 \mathrm{mg} /$ day (UCB, Brussels, Belgium), 1 used a combination of cetirizine hydrochloride and pseudoephedrine hydrochloride $5 \mathrm{mg}+120 \mathrm{mg} /$ day (UCB, Brussels, Belgium), and 4 used a combination of acrivastine and pseudoephedrine hydrochloride $8 \mathrm{mg}+60 \mathrm{mg} /$ once or twice a day (Glaxosmithkline).

\section{Sinus surgery}

Patients underwent endoscopic sinus surgery as previously described ${ }^{(17,18)}$. Briefly, randomized, standardised uncinectomy alone and uncinectomy with additional middle meatal antrostomy were performed on each side of each patient. Uncinecomy was performed similarly on both sides. The patient was blinded for the procedures performed on each side. On the antrostomy side, the size of the natural ostium was duplicated in the posterior direction by using cutting forceps. If necessitated, a large ethmoid bulla was opened on both ostium-preserving and enlarging sides in 25 patients. Posterior ethmoidal cells, sphenoidal sinuses and frontal sinuses were left undisturbed.

\section{Computed tomography scans}

High resolution CT imaging of the nasal airways and paranasal sinuses was performed on a ProSpeed PLUS scanner (General Electric, Milwaukee, WI, USA) equipped with a helical CT having a tube voltage of $120 \mathrm{kV}$ and tube current of $200 \mathrm{~mA}$. The thickness of the coronal slices was $3 \mathrm{~mm}$ with no intervening gap, a field of view of $25 \mathrm{~cm}$ and matrix size of 512 . Reconstructions were acquired post examination and were 1 $\mathrm{mm}$ thick. The extent of the inflammatory disease in computed 
tomography scans of the paranasal sinuses was determined by using the Lund-Mackay staging for rhinosinusitis ${ }^{(10)}$. LM staging is based on a simple numeric score derived from the CT scan. Each sinus group (maxillary, frontal, sphenoidal, anterior ethmoidal, and posterior ethmoidal) is assigned a numeric grade: $0=$ no abnormality, $1=$ partial opacification, and 2 $=$ total opacification. The ostiomeatal complex is scored as 0 (not obstructed) or 2 (obstructed). Thus a total score of 0 to 24 is possible, and each side can be considered separately ( 0 to 12). Analysis of the preoperative and postoperative CT scans and the staging of the CT scans were done on one occasion by two blinded authors (JPM and SKT-S).

The ostiomeatal complex was reconstructed with $1 \mathrm{~mm}$ slice thickness. The anteroposterior (AP) and cephalocaudal (CC) dimensions of the ostium were calculated by two blinded authors (JPM and PD) from the distance measurement data on the postoperative CT scan database. The maxillary sinus ostium was considered to be an ellipse with AP and CC dimensions as major and minor axes respectively. Thus the postoperative ostium size was determined to be $0.25 \pi \mathrm{APCC}$.

\section{Patient questionnaires}

The symptoms questionnaires were filled at one month preoperatively and at one, nine months and three years postoperatively. In this study we used the questionnaires filled at nine months postoperatively. We used total symptom score that was the sum of the following questions: facial pain/pressure (min-max 0-3), nasal obstruction (0-3), nasal discharge (0-3), posterior nasal drip (0-2), number of acute purulent sinusitis during the past 12 months $(0=$ zero, $1=$ one or two, $2=$ three or more), and sense of smell (0-3). Total symptom score may thus range from 0 to 16 , with lower scores representing better outcomes.

\section{Statistical analyses}

Statistics were performed with the SPSS Base 11.0 Statistical Software Package (SPSS, Chicago, IL, USA). Data is expressed as median and interquartile ranges. The nonparametric Wilcoxon rank sum test was used for comparison of matched pairs. The nonparametric spearman rank correlation test was used to study the correlations. The nonparametric Mann Whitney U test was used for comparisons of median or mean values in two groups. A two-tailed p-value of less than 0.05 was considered significant in all tests.

\section{RESULTS}

Observation of both sides of each CRS patient before operation revealed no significant differences statistically in the LM scores ( $p>0.05$, Wilcoxon test, Figure 1) or LM values for maxillary sinus opacification (0-2) or values for ostiomeatal complex obstruction $(0$ or 2$)(p>0.05$, Wilcoxon test, data not shown). Therefore, any postoperative differences in CT-parameters between the sides were considered to be due to the difference between ostium-preserving and enlarging techniques.
Comparison of the LM scores to CT scans taken prior to and 9 months postoperatively exposed a statistically significant difference on both ostium enlarging and preserving sides ( $p<0.001$, Wilcoxon test, Figure 1). Similarly, a significant improvement was achieved in postoperative LM values for maxillary sinus opacification and values for ostiomeatal complex obstruction on both sides $(\mathrm{p}<0.001$, Wilcoxon test, data not shown). In contrast, when comparing both sides, no statistically significant difference was observed in the postoperative LM scores ( $p>0.05$, Wilcoxon test, Figure 1) or in postoperative LM values for maxillary sinus opacification and values for ostiomeatal complex obstruction ( $\mathrm{p}>0.05$, Wilcoxon test, data not shown).

When observing both sides from postoperative CT scans, the anteroposterior (AP) diameter of the antrostomy side was statistically significantly greater than that of the uncinectomy side (mean values $0.98 \mathrm{~cm}, 0.52 \mathrm{~cm}$ respectively, $\mathrm{p}<0.001$, Wilcoxon test, Figure 2). Similar significant differences were found for the cephalocaudal (CC) diameter from postoperative CT-scans (mean values $0.75 \mathrm{~cm}, 0.41 \mathrm{~cm}$, respectively, $\mathrm{p}<0.001$, Wilcoxon test, Figure 3). Moreover, the ostium area was also significantly greater on the antrostomy side in calculations from postoperative CT scans (mean values $0.70 \mathrm{~cm}^{2}, 0.23$ $\mathrm{cm}^{2}$, respectively, $\mathrm{p}<0.001$, Wilcoxon test, data not shown).

A correlation was observed between the postoperative ostium area and postoperative change in LM values for maxillary sinus opacification and ostiomeatal complex obstruction ( $\mathrm{p}$ $<0.01, r=0.59$, Spearman rank correlation test, Figure 4). However, the postoperative symptoms/symptom sum did not correlate to any of the postoperative CT-values: postoperative size of ostium, postoperative LM score, change in LM score or change in LM values for maxillary sinus/ostiomeatal complex ( $p>0.05$, Spearman rank correlation test, data not shown).

The age, sex, or patient history of allergic rhinitis and/or asthma diagnosis, hypertrophic polypoid sinus mucosa, smoking, or intranasal corticosteroid and/or antihistamine medication, or the additional opening of the ethmoidal bulla, did not associate with the median values of the pre- or postoperative LM scores from either ostium preserving or enlarging side $(p>0.05$, by Mann Whitney U test, data not shown). Similarly, these parameters did not associate with the mean values of postoperative ostium area from either ostium-preserving or enlarging side ( $\mathrm{p}$ $>0.05$, by Mann Whitney U test, data not shown).

\section{DISCUSSION}

This study was performed to compare two operative techniques in endoscopic sinus surgery: uncinectomy vs. uncinectomy with middle meatal antrostomy. The comparison was made by observing Lund-Mackay scores and ostium size prior to and 9 months after the operation. The correlation between the postoperative CT findings and subjective outcomes was studied in order to evaluate if larger ostium size or low LM score indicate better symptom relief. 


\begin{tabular}{|c|c|c|c|c|c|c|}
\hline Patient & Age & $\begin{array}{l}\text { Patient } \\
\text { history }\end{array}$ & Smoking & Medication & LM-score & $\begin{array}{l}\text { Postop. area of } \\
\text { ostium }\left(\mathrm{cm}^{2}\right)\end{array}$ \\
\hline female $^{\dagger}$ & 43 & CRS & + & $\mathrm{A} /-$ & $5,5 /-,=$ &,$-=$ \\
\hline female & 64 & CRS & - & $-/-$ & $5, \underline{8} / 2, \underline{3}$ & $0.65, \underline{1.05}$ \\
\hline female & 60 & CRS & - & $\mathrm{C} / \mathrm{C}$ & $\underline{5}, 5 / 2,4$ & $\underline{0.19}, 0.00$ \\
\hline female & 22 & CRS & - & $\mathrm{C} / \mathrm{C}$ & $5, \underline{5} / 5, \underline{3}$ & $0.00, \underline{0.88}$ \\
\hline female & 42 & CRS & - & $-/-$ & $\underline{5}, 5 / \underline{4}, 4$ & $\underline{0.52}, 0.26$ \\
\hline male & 52 & CRS & - & $\mathrm{C} / \mathrm{C}$ & $\underline{6}, 6 / \underline{3}, 3$ & $\underline{0.16}, 0.12$ \\
\hline female & 40 & $\mathrm{CRS}, \mathrm{NP}^{*}$ & + & $-/-$ & $5, \underline{5} / 5, \underline{2}$ & $0.00, \underline{1.06}$ \\
\hline female & 37 & CRS,HP & - & $-/-$ & $5, \underline{5} / 1, \underline{1}$ & $0.57, \underline{1.22}$ \\
\hline female & 40 & CRS,HP & + & $-/-$ & $\underline{7}, 7 / \underline{2}, 2$ & $\underline{0.20}, 0.10$ \\
\hline male & 42 & CRS,HP,AR & - & $-/-$ & $\underline{3}, 3 / 2,2$ & $\underline{0.06}, 0.07$ \\
\hline female & 50 & CRS,HP,AR & - & $\mathrm{C} /-$ & $5, \underline{5} / 5, \underline{2}$ & $0.00, \underline{0.28}$ \\
\hline female & 42 & CRS,HP,AR & - & $\mathrm{C} / \mathrm{C}$ & $5, \underline{5} / 0, \underline{2}$ & $0.21, \underline{0.13}$ \\
\hline female & 53 & CRS,HP,AR,A & - & $-/-$ & $5, \underline{5} / 2, \underline{2}$ & $0.33, \underline{1.01}$ \\
\hline female & 49 & CRS,HP,AR,A & - & $\mathrm{AC} / \mathrm{AC}$ & $\underline{6}, 6 / \underline{3}, 3$ & $\underline{0.19}, 0.05$ \\
\hline male & 58 & CRS,HP,A & - & $-/ \mathrm{C}$ & $\underline{7}, 7 / \underline{3}, 3$ & $\underline{1.00}, 0.20$ \\
\hline male & 31 & CRS,HP,A & - & $-1-$ & $\underline{4}, 4 / \underline{4}, 2$ & $\underline{0.00}, 0.19$ \\
\hline male & 56 & CRS,AR & + & $-1-$ & $4, \underline{4 / 0, \underline{0}}$ & $0.41, \underline{0.95}$ \\
\hline female & 55 & CRS,AR & - & $-1-$ & $5, \underline{5} / 2, \underline{2}$ & $0.66, \underline{0.47}$ \\
\hline male & 52 & CRS,AR & - & $-1-$ & $6, \underline{5} / 2, \underline{2}$ & $0.02, \underline{0.88}$ \\
\hline male & 46 & CRS,AR & - & $-1-$ & $5, \underline{5} / 3, \underline{3}$ & $0.05, \underline{0.42}$ \\
\hline male & 30 & CRS,AR & + & $\mathrm{A} / \mathrm{A}$ & $\underline{5}, 5 / \underline{1}, 1$ & $\underline{1.75}, 0.08$ \\
\hline female & 62 & CRS,AR & + & $-/-$ & $\underline{4}, 4 / \underline{0}, 0$ & $\underline{1.10}, 0.83$ \\
\hline female & 38 & CRS,AR & - & $\mathrm{AC} / \mathrm{AC}$ & $\underline{4}, 4 / \underline{2}, 1$ & $\underline{0.61}, 0.14$ \\
\hline female & 21 & CRS,AR & - & $\mathrm{AC} /-$ & $5, \underline{6} / 1, \underline{1}$ & $0.55, \underline{1.45}$ \\
\hline female & 45 & CRS,AR,A & - & $-1-$ & $\underline{5}, 5 / \underline{2}, 2$ & $\underline{0.15}, 0.14$ \\
\hline male & 58 & CRS,AR,A & - & $\mathrm{C} /-$ & $\underline{4}, 5 / \underline{1}, 2$ & $\underline{1.12}, 0.61$ \\
\hline female & 30 & CRS,AR,A & + & $-/-$ & $5, \underline{5} / 5, \underline{2}$ & $0.00, \underline{2.09}$ \\
\hline male & 62 & CRS,A & + & $-/-$ & $\underline{7}, 6 / \underline{1}, 1$ & $\underline{0.01}, 0.01$ \\
\hline female & 66 & CRS,A & - & $\mathrm{C} / \mathrm{C}$ & $\underline{5}, 6 / \underline{2}, 2$ & $\underline{0.99}, 0.28$ \\
\hline female & 30 & CRS,A & - & $\mathrm{AC} / \mathrm{AC}$ & $3, \underline{5} / 1, \underline{1}$ & $0.26, \underline{0.36}$ \\
\hline
\end{tabular}

Table 1. The characteristics of the patient groups used in this study. The age (years) of the patients is shown at the time of operation. Abbreviations: Patient history: $\mathrm{Co}=$ control patient, $\mathrm{AR}=$ allergic rhinitis, $\mathrm{CRS}=$ chronic rhinosinusitis, $\mathrm{NP}=$ nasal polyposis, $\mathrm{A}=$ asthma. $\mathrm{NP} *$ indicates postoperative nasal polyposis with one exceptional case. Medication: $\mathrm{A}=$ antihistamine, $\mathrm{C}=$ intranasal corticosteroid. The backslash $(/)$ between the letters indicates preoperative / postoperative medication. LM-score: Lund-Mackay score for computed tomography (CT) scans. The backslash (/) separates preoperative / postoperative CT-scans. The comma (,) separates the right and left side of the sinonasal tract. The underline (_) stands for the side on which middle meatal antrostomy was performed. Postop. area of ostium $\left(\mathrm{cm}^{2}\right)$ : The area (in square centimeters) is counted from the CT-scans taken 9 months postoperatively. The comma (,) separates the right and left side of the sinonasal tract. The underline (_) stands for the side on which middle meatal antrostomy was performed. $\uparrow=$ the patient died accidentally before the last follow-up. Thus the patient was withdrawn from the analysis. 


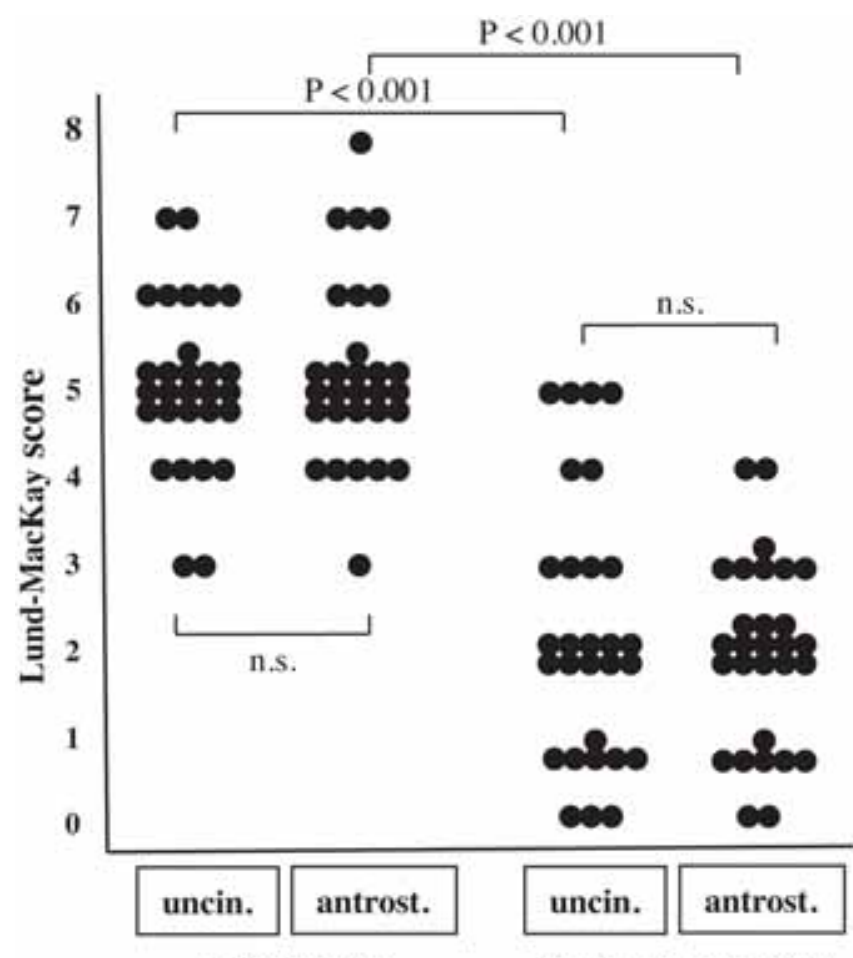

preoperative

9 mo. postoperative
Figure 1. Comparison of the unilateral Lund-Mackay (LM) scores for computed tomography scans taken preoperatively and 9 months postoperatively in patients who had undergone uncinectomy on one side and additional middle meatal antrostomy on the other side. There was a significant decrease in the postoperative LM scores for computed tomography scans in comparison to preoperative LM scores (by Wilcoxon test). When comparing uncinectomy and antrostomy sides with each individual, there was not a significant difference (n.s.) between these sides in either preoperatively or 9 months postoperatively taken computed tomography scans (by Wilcoxon test).

Others have also compared uncinectomy and additional middle meatal antrostomy on each side of each CRS patient ${ }^{(19)}$. In contrast to our study, the patients had additional nasal polyps and the CT-findings were not evaluated. Wadwongtham et al., found in this prospective randomized study that a large middle meatal antrostomy had a better patency rate than an undisturbed maxillary sinus ostium only in the early phase of evaluation (3 months postoperatively). From the 6th month to the final evaluation (1 year) there was no statistically significant difference between the surgical techniques, recurrent polyposis being the main reason for re-occclusion of the drainage system ${ }^{(19)}$.

Although clinical studies have shown that Lund-Mackay scores have little correlation in symptom severity, it is still the most widely used radiological method for assessing the diagnosis and the severity of CRS ${ }^{(9,13)}$. We showed that both the preserving and enlarging techniques occasioned a significant reduction of the LM scores. This is in accordance with the previous observations for the effect of ESS on the LM scores ${ }^{(14)}$.

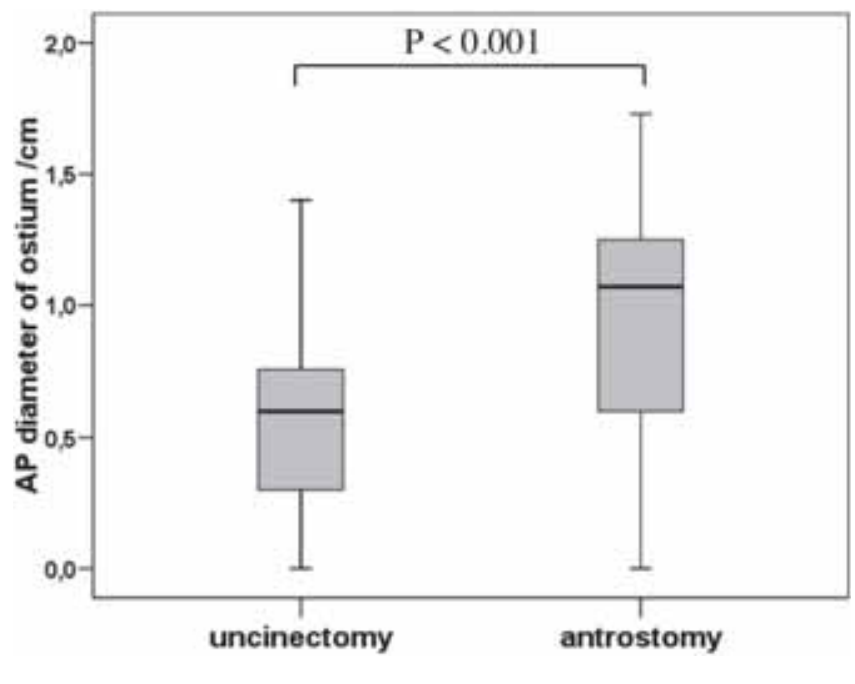

Figure 2. The y-axis represents the anterior-posterior (AP) diameter of the maxillary sinus ostium calculated from the computed tomography scans taken 9 months postoperatively in patients who had undergone uncinectomy on one side and additional middle meatal antrostomy on the other side. The AP-diameter of the ostium remained greater on the antrostomy side than on the uncinectomy side 9 months postoperatively (by Wilcoxon test).

On one hand, measurements from the postoperative CT scans showed that the diameter of the ostium remained greater on the antrostomy side than on the ostium preserving side. On the other hand there was a correlation between the postoperative ostium area and postoperative change in LM values for maxillary sinus opacification and ostiomeatal complex obstruction. Thus, an uncinectomy with additional middle meatal antrostomy seemed to be associated with lower LM scores than uncinectomy without antrostomy.

We have previously shown with these subjects, that the number of eosinophils in the sinus mucosa decreased only on the side on which the ostium was enlarged, not on ostium preserving side ${ }^{(18)}$. Thus, antrostomy might normalize more effectively not only the severity of the mucosal inflammation but also the volume of the inflamed sinus mucosa. Yet, because we were not able to show that achieving lower LM scores by larger ostium size provided superior symptom relief, additional middle meatal antrostomy might not be expected to be more effective than simple uncinectomy only. However, further studies with larger numbers of patients are required to study this. 


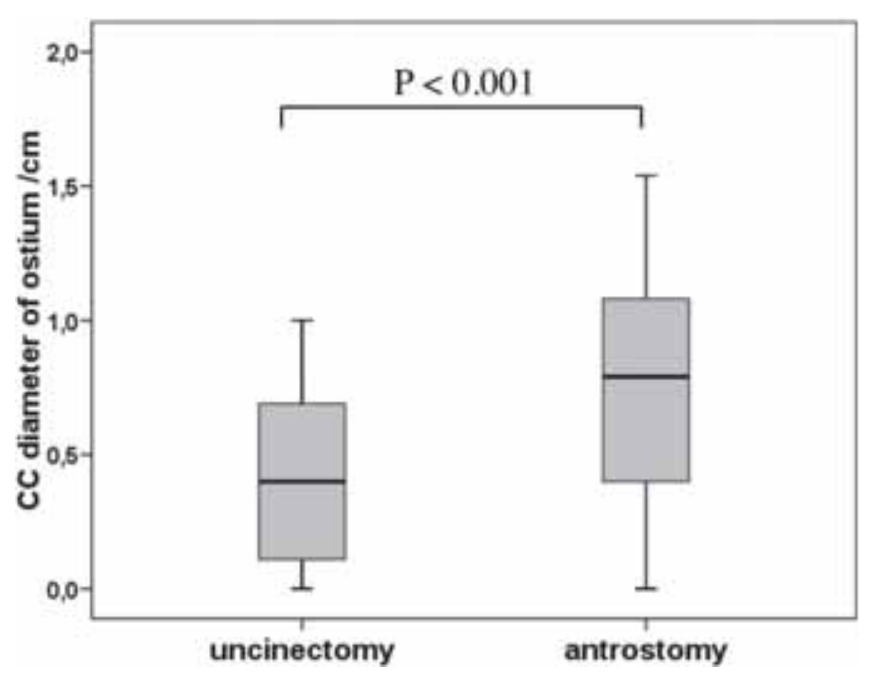

Figure 3. The y-axis represents the cephalocaudal (CC) diameter of the maxillary sinus ostium calculated from the computed tomography scans taken 9 months postoperatively in patients who had undergone uncinectomy on one side and additional middle meatal antrostomy on the other side. The CC-diameter of the ostium remained greater on the antrostomy side than on the uncinectomy side 9 months postoperatively (by Wilcoxon test).

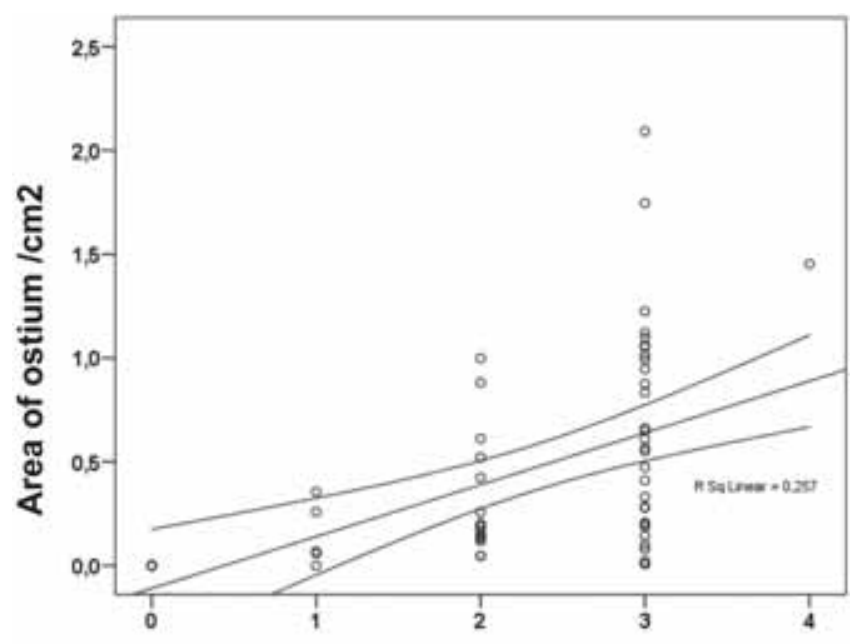

Change in Lund-Mackay Max.sinus+omc

Figure 4. The correlation between the area of the maxillary sinus ostium and the unilateral postoperative change in LundMackay (LM) values for maxillary sinus opacification plus ostiomeatal complex obstruction (= Max.sinus+OMC). The values were evaluated from the computed tomography scans taken 9 months postoperatively. The area was calculated from the mathematical equation $0.25 \pi \mathrm{APCC}$. Increased postoperative area of the maxillary sinus ostium correlated with greater postoperative change in LM values for maxillary sinus opacification and ostiomeatal complex obstruction (by Spearman rank correlation test). $r=$ correlation coefficient.

By using the reconstructed $1 \mathrm{~mm}$ CT slice thickness of the ostiomeatal complex, we were able not only to calculate the ostium area, but also to show that there was no significant postopera- tive scarring or adhesion formation on either side. This might thus, indicate good recovery of the ostiometal complex with both procedures.

Measurement methods, such as SinoNasal outcome Test-20, 36-Item Short-Form health Survey, and Visual Analogue Score, to analyze symptom outcomes after ESS were not used in this study as conception work began in 2000, when standardized Quality of Life methods were not in general use. We acknowledge the fact that this makes comparison to other studies difficult.

\section{CONCLUSION}

The postoperative decrease of the Lund-McKay scores on both sides seems to indicate the good outcomes of ESS, regardless of the type of procedure performed. When observing the postoperative postoperative change in LM values for maxillary sinus opacification and ostiomeatal complex obstruction, an uncinectomy with additional antrostomy seems to be slightly more effective than uncinectomy without antrostomy. However, these CT findings did not associate to symptoms asked postoperatively. Thus, uncinectomy with additional middle meatal antrostomy seems to have no benefit over simple uncinectomy.

\section{ACKNOWLEDGEMENTS}

We thank research nurse Marja-Leena Oksanen for excellent collaboration. Source of financial support: supported in part by research grants from the Medical Research Fund of PäijätHäme Central Hospital, Medical Research Fund of Tampere University Hospital, the Finnish Society of Allergology and Immunology, and the Finnish Society of Otorhinolaryngology.

\section{REFERENCES}

1. Fokkens W, Lund V, Bachert C, et al. EAACI: EAACI position paper on rhinosinusitis and nasal polyps. Rhinology. 2007; (Suppl. 20): 1-136.

2. Senior BA, Kennedy DW, Tanabodee J, Kroger H, Hassab M, Lanza D. Long-term results of functional endoscopic surgery. Laryngoscope. 1998; 108: 151-157.

3. Khalid AN, Quraishi SA, Kennedy DW. Long-term quality of life measures after functional endoscopic sinus surgery. Am J Rhinol. 2004; 18: 131-136.

4. Kennedy DW. Functional endoscopic sinus surgery. Technique. Arch Otolaryngol 1985; 111: 643-649.

5. Catalano PJ, Strouch M. The minimally invasive sinus surgery: theory and practice. Otolaryngol Clin North Am. 2004; 37: 401-409.

6. Setliff RC III. Minimally invasive sinus surgery: the rationale and technique. Otolaryngol Clin North Am. 1996; 29: 115-124.

7. Albu S, Tomescu E. Small and large middle meatal antrostomies in the treatment of chronic maxillary sinusitis. Otolaryngol Head and Neck Surg. 2004: 131: 542-547.

8. Catalano P, Roffman E. Outcome in patients with chronic sinusitis after the minimally invasive sinus technique. Am J Rhinol. 2003; 17: 17-22.

9. Salama N, Oakley RJ, Skilbeck CJ, Choudhury N, Jacob A. Benefit from minimally invasive sinus technique. J Laryngol Otol. 2009; 123: 186-90.

10. Lund VJ, Mckay IS. Staging in rhinosinusitis. Rhinology. 1993; 107: 183-184.

11. Smith TL, Batra PS, Seiden AM, Hannley M. Evidence supporting endoscopic sinus surgery in the management of adult chronic rhinosinusitis: a systemic rewiew. Am J Rhinolol. 2005; 19: 537-543. 
12. Holbrook EH, Brown CL, Lyden ER, Leopold DA. Lack of significant correlation between rhinosinusitis symptoms and specific regions of sinus computer tomography scans. Am J Rhinol. 2005; 19: 382-387.

13. Hopkins C, Brown JP, slack R, Lund V, Brown P. The LundMacKay staging system for chronic rhinosinusitis: How is it used and what does it predict? Otolaryngol Head Neck Surg. 2007; 131: 555-561.

14. Sharp HR, Rowe-Jones JM, McKay IS. The outcome of endoscopic sinus surgery: Correlation with computerized tomography, score and systemic disease. Clin Otolaryngol. 1999; 24: 39-42.

15. Messerklinger W. Endoscopy of the nose. Baltimore, MD: Urban and Scwarzenberger; 1978.

16. Stammberger H. Endoscopic endonasal surgery-concepts in treatment of recurring rhinosinusitis. Part I. Anatomic and pathophysiological considerations. Otolaryngol Head Neck Surg. 1986; 94: 143-147.

17. Myller J, Toppila-Salmi, Torkkeli T, Heikkinen J, Rautiainen M. Effect of endoscopic sinus surgery on antral mucociliary clearance. Rhinology. 2006; 44: 193-196.

18. Myller JP, Toppila-salmi SK,Toppila EM, Torkkeli TV, Renkonen
RL, et al. Mucosal eosinophils and L-selectin liagands are associated with invasive and non-invasive sinus surgery outcomes. Am J Rhinol Allergy. 2009; 23: 21-27.

19. Wadwongtham W, Aeumjaturapat S. Large middle meatal antrostomy vs undisturbed maxillary ostium in the endoscopic sinus surgery of nasal polyposis. J Med Assoc Thai. 2003; 86, Suppl 2: S373-378.

Jyri Myller, M.D.

Päijät-Häme Central Hospital

Keskussairaalankatu 7

15850, Lahti

Finland

Tel: +358-3-819-2312

Fax: +358-3-819 2049

E-mail address: jyri.myller@phsotey.fi

\section{9th INTERNATIONAL COURSE IN ADVANCED SINUS SURGERY TECHNIQUES}

\section{Dissection course with fresh frozen cadaver heads Teacher of Honour: Prof. Manuel Bernal Sprekelsen}

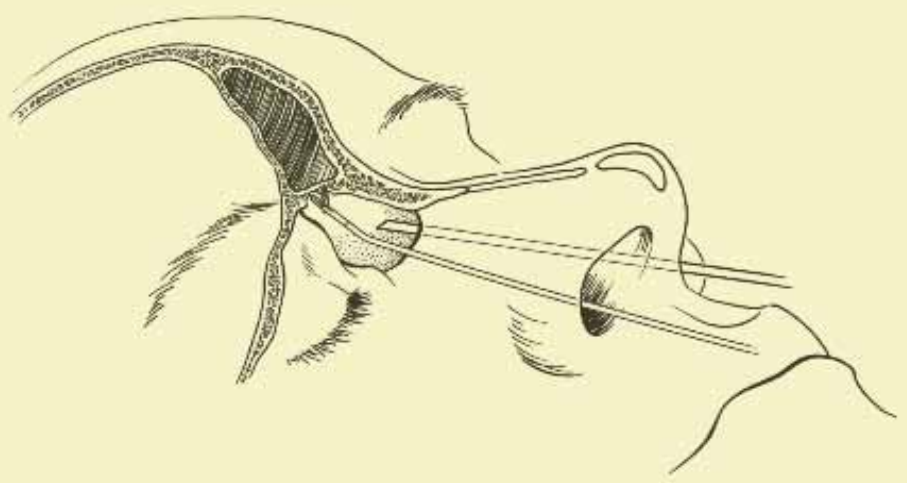

March 22-23 March, 2012

Department of Otorhinolaryngology

Academic Medical Center of the University of Amsterdam The Netherlands 